\title{
Development of the Thirty-Meter Telescope project
}

\author{
Edward Stone $^{1}$ and Michael Bolte ${ }^{2, *}$ \\ ${ }^{1}$ Division of Physics, Mathematics and Astronomy, California Institute of Technology Pasadena, CA, USA \\ ${ }^{2}$ Department of Astronomy and Astrophysics, University of California, Santa Cruz, Santa Cruz, CA, USA
}

\begin{abstract}
The Thirty-Meter Telescope (TMT) project is a welladvanced effort to construct one of the first of the next generation of extremely large telescopes with primary mirror diameter larger than $25 \mathrm{~m}$. When used with an adaptive optics systems capable of producing diffraction-limited images, the science reach of a $30 \mathrm{~m}$ telescope is extraordinary with improvements in sensitivity over existing telescopes by as much as a factor of 80 for some kinds of observations. The TMT design extends that of the twin Keck $10 \mathrm{~m}$ telescopes now in operation and uses the same close-packed segmented primary mirror. The partnership that will finish the design, construct and operate the telescope is described.
\end{abstract}

Keywords: Adaptive optics system, large telescopes, segmented mirrors.

\section{Introduction}

BECAUSE the vast majority of objects in the Universe are at enormous distances from the Earth, they are faint in terms of the flux of electromagnetic radiation and appear small in angular size. For these reasons the continuing development of telescopes and the instrumentation used with them have been key to the last 400 years of discovery of the contents, physical laws governing, and the very nature of the Universe. When Galileo first observed celestial objects using a small telescope, he improved upon the previous observations of humankind using the unaided eye in two ways. With a larger collecting area compared to the unaided, eye he could observe fainter objects and with the higher resolving power he could see finer details. For the first time, the phases of Venus could be discerned, the moons of Jupiter detected and the Milky Way resolved into thousands of individual stars. These improvements in the quality of astronomical observations led to a revolution in our view of the Universe and the place of the Earth and Solar System in it.

In the years since Galileo, telescopes have gotten larger and their performance better. In the late 1800s, a transition was made from refracting telescopes utilizing lenses to gather light to reflecting telescopes that use mirrors.

*For correspondence. (e-mail: bolte@ucolick.org)
This was driven primarily by the goal of ever-larger collecting areas. Lenses have to be supported at their perimeter to avoid blockage, while mirrors can be supported from the back. During the era when the existence of the extra-galactic universe was established, the expansion of the Universe discovered, the verification of general relativity was made and the basic processes of stellar evolution became understood, telescopes grew from $1 \mathrm{~m}$ class to the $5 \mathrm{~m}$ Palomar Observatory Hale telescope and each improvement in telescope, technology and size led directly to many of the science advances.

The Palomar $5 \mathrm{~m}$ telescope had first light in 1949 and remained the most productive instrument (a Soviet Union 6-metre telescope was larger, but performed poorly) for more than 40 years. The pause in the development of larger primary mirrors was due to the difficulty in maintaining the exquisite surface figure of the mirror in the face of a changing gravity vector as the telescope was moved to track different objects in the sky. The $5 \mathrm{~m}$ mirror weighs 15 tonnes and the complex, adaptive mechanical support system attached to the back of the mirror weighs almost as much.

\section{Segmented mirrors and adaptive optics}

Two new technologies developed in the 1980s have proven key to developing telescopes with primary mirrors significantly larger than the $5 \mathrm{~m}$ Palomar and key to the science case for building significantly larger primary mirrors in telescopes. The key technology that allows for significantly larger effective primary size was the development in the 1980s of segmented primary mirrors by Jerry Nelson, Terry Mast and collaborators at the University of California, USA. The segmented mirror technology was first proven in a working telescope with the Keck 1 telescope at the W.M Keck Observatory in 1990. This Observatory was developed, constructed and is operated by a partnership of the University of California and the California Institute of Technology (Caltech).

The Keck telescope primary is composed of 36 hexagonal segments that are arranged in a close-packed configuration with sensors and actuators that can measure and move segments with respect to one another at a precision of a few nanometres. The entire array of sensor 
values is used to solve for the actuator settings that allow the 36 segments to act as a single, high-precision surface with a collecting area of $10 \mathrm{~m}$. The Keck Telescope segments are $1.8 \mathrm{~m}$ in diameter (circumscribed circle) and only $7.5 \mathrm{~cm}$ thick. The correction bandwidth is $2 \mathrm{~Hz}$. Each of the components of the segmented primary mirror required significant new technology developments ${ }^{1-3}$. Because the individual segments are small, they can be much thinner than a large monolithic mirror, and the weight of all the segments can be much smaller than the equivalent diameter monolithic mirror, greatly simplifying the telescope structure needed to support and control the mirror shape.

The second new technology that directly impacts the motivation to build larger primary mirrors is the development of adaptive optics (AO) that can be used to measure and correct for atmospheric disturbances above a telescope. The highest resolution that can be achieved with a given primary aperture is set by the 'diffraction limit' of the mirror. The diffraction limit for a telescope primary is given by

$$
\theta=\frac{1.22 \times \lambda(\mathrm{cm})}{D(\mathrm{~cm})}
$$

where $\lambda$ is the wavelength of light, $D$ the diameter of the primary mirror and $\theta$ is measured in radians. A $30 \mathrm{~m}$ primary could in theory deliver images three times sharper than a $10 \mathrm{~m}$ mirror and 12.5 times sharper than the $2.4 \mathrm{~m}$ Hubble Space Telescope primary mirror. At a wavelength of $1 \mu \mathrm{m}$, the $30 \mathrm{~m}$ diffraction limit is 0.007 arcsec.

However, even at a very good ground-based site, atmospheric blurring limits resolution to around 0.5 arcsec. For the first 400 years of increasingly larger telescopes, the principal benefit of larger mirrors was greater light-gathering capability because atmospheric blurring made it impossible to take advantage of the finer diffraction limit of the larger primary mirrors. This changed with the development of AO systems. With a sufficiently capable AO systems, it is possible to correct for the blurring and focus a significant fraction of the light gathered by the primary mirror into the diffractionlimited Airy disk. For current generation AO systems at a good observatory site, it is possible to correct to the diffraction limit of a primary of $10 \mathrm{~m}$ diameter (ref. 4).

The advantages of working at the diffraction limit of a large telescope are significant. For some science, being able to resolve finer details is crucial. For backgroundlimited observations (faint sources compared to the sky or other background light) of point sources, the amount of background radiation is reduced by the square of the diffraction limit. For this case, sensitivity increases as the primary diameter to the fourth power when including the greater light-gathering capability and reduced backgrounds. Using AO, it is possible to design spectrometers with high spectral resolution based on a narrow slit which reduces dramatically the overall size of the instrument and associated optics.

The twin Keck Observatory 10-metre telescopes demonstrated that segmented mirror technology can be made to work routinely and reliably in an observatory environment. The development of a highly-effective AO system at the Keck telescopes showed that the segments can be aligned and phased well enough to produce diffractionlimited images in the focal plane. The Keck-1 telescope has now been in operation for 24 years and a number of telescopes in the $10 \mathrm{~m}$ class have been in operation around the world for the last decade. Scientists have been pushing the limits of large telescope astronomy in many different areas. As the Keck telescopes were under development, it was recognized that the segmented mirror technology was intrinsically scalable. To make a larger mirror required only adding additional rings of segments, and a number of groups initiated studies starting in the early 2000s to build a new-generation telescope in the 30-metre class.

\section{Extremely large telescopes}

The Thirty-Meter Telescope (TMT) project is one of three projects currently in the advanced stage of design. In large part because of the involvement of the University of California and Caltech, the TMT follows most closely the Keck telescope designs. The primary mirror is composed of 492 hexagonal segments, each $1.4 \mathrm{~m}$ in diameter and the telescope optical design is a Ritchey-Chretien with large Nasmyth platforms for instruments. The primary mirror is $f / 1$ and the focal ratio at the platforms is f/15. The fast primary ratio reduces the size of the telescope and dome significantly compared to a scaled-up Keck telescope and dome. The smaller segments of the TMT compared to the Keck telescopes $(1.8 \mathrm{~m})$ are a trade-off between the ease of figuring segments which increases with decreasing segment size, and the added complexity of additional sensors and actuators for smaller segments at a given primary mirror size.

The TMT project started in 2004 with the merger of three previously independent investigations. The $30 \mathrm{~m}$ diameter was chosen after serious consideration as the best balance of technology, and cost risk and advancement of scientific capabilities. At three times the diameter of the currently largest optical-IR telescopes, this would be the largest step forward in capability since Galileo's first use of a telescope. One important consideration was that a $30 \mathrm{~m}$ telescope requires a more capable AO system that the current $10 \mathrm{~m}$ telescopes; so such a system is now under development and will be available when the telescope begins operations.

The TMT partnership developed over a decade. The initial members of the partnership in 2004 were the Association of Canadian Universities for Research in 
Astronomy (ACURA), the Association of Universities for Research in Astronomy (AURA), Caltech, and the University of California. AURA dropped out in 2007. The project underwent a series of intensive reviews and made a number of critical design decisions. Partners were added first as Observers then as Participants in the project with decisions being taken by a 'collaborative board' of participants. Between 2011 and 2014, the Participants worked intensively on a Partnership Agreement. In 2014, this resulted in the formation of the TMT International Observatory Limited Liability Company (TIO). The TIO Members as of January 2017 are Caltech; the National Research Council of Canada; the Chinese Academy of Sciences, the Department of Science and the Technology and Department of Atomic Energy, Government of India, the National Institutes of Natural Sciences Japan, and the University of California. AURA, on behalf of the US at-large astronomy community is an Associate of TIO.

The project is being developed with major technology contributions from each of the Member partners. Partner deliverables and the resulting shares in TIO are described in the TIO Workshare Matrix. For example, India is responsible for the primary mirror segment support and control hardware, a portion of the segment fabrication, observatory software and instrumentation ${ }^{5}$. The arrangements for contributions to the project are that up to $70 \%$ of a Member contribution can be in the form of in-kind contributions as defined in the Workshare Matrix and the remainder of the contribution in cash to the TIO Project office to cover overall management of the project and certain common infrastructure for the construction project.
After having received permits and legal access to the selected site on Mauna Kea in Hawaii in 2014, the project has been delayed by complications with access. In January 2017, a part of the initial permitting process was redone in Hawaii with hearings that are expected to be concluded by early Spring 2017. For the case that the Hawaii process does not result in a re-issuance of a permit in a timely way, the project is working through the permitting process and establishing agreements with the Institutio de Astrofisica de Canarias which operates the Observatorio del Roque de los Muchachos on the island of La Palma in the Canary Islands. This provides a back-up site with excellent conditions for observations and retains access to the northern hemisphere sky.

At Mauna Kea or La Palma, the TIO Board has established a schedule for initiation of on-site construction no later than spring 2018.

1. Nelson, J. E., Stressed mirror polishing: fabrication of an off-axis section of a paraboloid. Appl. Opt., 1980, 19, 23412352.

2. Mast, T. S. and Nelson, J. E., Figure control for a fully segmented telescope mirror. Appl. Opt., 1982, 21, 2631-2641.

3. Mast, T. S., Gabor, G. and Nelson, J. E., Edge sensors for a segmented mirror. Proc. SPIE, 1983, 444, 297-309.

4. Wizinowich, P. L. et al., The W. M. Keck Observatory laser guide star adaptive optics system: overview. Pub. Astr. Soc. Pac., 2006, 118, 297-309.

5. Reddy, B. E., India's participation in the Thirty-Meter Telescope project, J. Astrophys. Astron., 2013, 34, 87-95.

doi: 